\title{
Gemcitabine diphosphate choline is a major metabolite linked to the Kennedy pathway in pancreatic cancer models in vivo
}

\author{
T E Bapiro ${ }^{1}$, K K Frese ${ }^{1,3}$, A Courtin ${ }^{1,4}$, J L Bramhall ${ }^{1}$, B Madhu ${ }^{1}$, N Cook ${ }^{1,3}$, A Neesse ${ }^{1,5}$, J R Griffiths ${ }^{1}$, \\ D A Tuveson ${ }^{2}$, D I Jodrell ${ }^{\star, 1}$ and F M Richards ${ }^{\star, 1}$ \\ ${ }^{1}$ Cancer Research UK Cambridge Institute, University of Cambridge, Li Ka Shing Centre, Box 278, Robinson Way, Cambridge \\ CB2 ORE, UK and ${ }^{2}$ Cold Spring Harbor Laboratory, 1 Bungtown Road, Cold Spring Harbor, NY 11724, USA
}

Background: The modest benefits of gemcitabine $(\mathrm{dFdC})$ therapy in patients with pancreatic ductal adenocarcinoma (PDAC) are well documented, with drug delivery and metabolic lability cited as important contributing factors. We have used a mouse model of PDAC: KRAS ${ }^{\mathrm{G} 12 \mathrm{D}}$; $\mathrm{p} 53^{\mathrm{R} 172 \mathrm{H}}$; pdx-Cre (KPC) that recapitulates the human disease to study dFdC intra-tumoural metabolism.

Methods: LC-MS/MS and NMR were used to measure drug and physiological analytes. Cytotoxicity was assessed by the Sulphorhodamine B assay.

Results: In KPC tumour tissue, we identified a new, Kennedy pathway-linked dFdC metabolite (gemcitabine diphosphate choline $(\mathrm{GdPC})$ ) present at equimolar amounts to its precursor, the accepted active metabolite gemcitabine triphosphate (dFdCTP). Utilising additional subcutaneous PDAC tumour models, we demonstrated an inverse correlation between GdPC/dFdCTP ratios and cytidine triphosphate (CTP). In tumour homogenates in vitro, CTP inhibited GdPC formation from dFdCTP, indicating competition between CTP and dFdCTP for CTP:phosphocholine cytidylyltransferase (CCT). As the structure of GdPC precludes entry into cells, potential cytotoxicity was assessed by stimulating CCT activity using linoleate in KPC cells in vitro, leading to increased GdPC concentration and synergistic growth inhibition after $\mathrm{dFdC}$ addition.

Conclusions: GdPC is an important element of the intra-tumoural $\mathrm{dFdC}$ metabolic pathway in vivo.

Gemcitabine (dFdC) has been the drug used most frequently for patients with pancreatic ductal adenocarcinoma (PDAC) for several decades, but its modest effects are well documented, with median survival of only 6.7 months (Von Hoff et al, 2013). Research has focused on drug delivery and metabolic lability as the potential causes for the poor response rates. Metabolic activation of $\mathrm{dFdC}$ in cells is initiated by deoxycytidine kinase (dCK) to give the monophosphate. Further sequential phosphorylation yields the diphosphate and triphosphate, both responsible for the cytotoxic effects of the drug. Gemcitabine diphosphate (dFdCDP) inhibits ribonucleotide reductase, which catalyses formation of deoxynucleotide triphosphates required for DNA synthesis. When incorporated into DNA, gemcitabine triphosphate (dFdCTP) terminates DNA chain elongation (Huang et al, 1991; Plunkett et al, 1995). $2^{\prime}, 2^{\prime}$-difluorodeoxyuridine (dFdU) has been considered to be the major inactive metabolite of $\mathrm{dFdC}$; however, its triphosphorylated form dFdUTP may contribute to cytotoxicity (Veltkamp et al, 2008). Metabolism-based approaches to improve $\mathrm{dFdC}$ efficacy are ultimately aimed at increasing the amount of dFdCTP and have thus far manipulated nodes in the drug's

*Correspondence: Professor DI Jodrell; E-mail: Duncan.Jodrell@cruk.cam.ac.uk or Dr FM Richards; E-mail: Fran.Richards@cruk.cam.ac.uk

${ }^{3}$ Current address: Princess Margaret Hospital, 610 University Avenue, Toronto M5G 2M9, Ontario, Canada.

${ }^{4}$ Current address: Astex Pharmaceuticals, 436 Cambridge Sciences Park Milton Road, Cambridge CB4 00A, UK.

${ }^{5}$ Current address: Department of Gastroenterology, Endocrinology and Metabolism, Philipps University Marburg, Baldingerstr, 35043 Marburg, Germany. 
metabolic pathway likely to favour dFdCTP formation, such as reducing cytidine deaminase-mediated $\mathrm{dFdU}$ formation (Beumer et al, 2008; Costanzi et al, 2011; Tibaldi et al, 2011). However, such approaches will be informed by knowledge of intra-tumoural metabolism of the drug that has been limited by the scarcity of tissue specimens from patients.

To gain insight into dFdC pharmacology, we recently developed an assay to measure $\mathrm{dFdC}$ and its metabolites in tumour tissue from the KRAS ${ }^{\mathrm{G} 12 \mathrm{D}}$; p53 ${ }^{\mathrm{R} 172 \mathrm{H}}$; pdx-Cre (KPC) genetically engineered mouse model of PDAC (Bapiro et al, 2011). Using the same assay, the likelihood of further metabolism of dFdCTP was also investigated, with a focus on its possible metabolism by enzymes in the Kennedy pathway of phosphatidylcholine (PtdCho) synthesis. The precedent for this is cytosine arabinoside (Ara-C) (Lauzon et al, 1978a,b), the diphosphate choline metabolite of which is thought to be formed from PtdCho and Ara-C monophosphate, in a reversal of the reaction catalysed by cholinephosphotransferase (CPT) (Figure 1; Kucera and Capizzi, 1992). PtdCho is a phospholipid with critical roles in lipid membrane synthesis and signal transduction. The majority of PtdCho is synthesised from choline via the Kennedy pathway in three steps (Figure 1) catalysed by the enzymes choline kinase (CK), CTP:phosphocholine cytidylyltransferase (CCT) and CPT. We hypothesised that dFdCTP could compete with the physiological Kennedy pathway substrate cytidine triphosphate (CTP) for the enzyme CCT, resulting in the formation of gemcitabine diphosphate choline (GdPC) in place of the normal physiological metabolite CDP choline.

Here, we report the identification of GdPC as a major $\mathrm{dFdC}$ metabolite in murine PDAC tumour tissue from the KPC genetically engineered mouse. In MIA PaCa-2 xenograft tissue the GdPC/dFdCTP ratio was lower than in KPC PDAC tissue, and in K8484 (KPC PDAC cell line) allograft tissue the GdPC/dFcCTP ratio was higher than in KPC PDAC. Therefore, using these

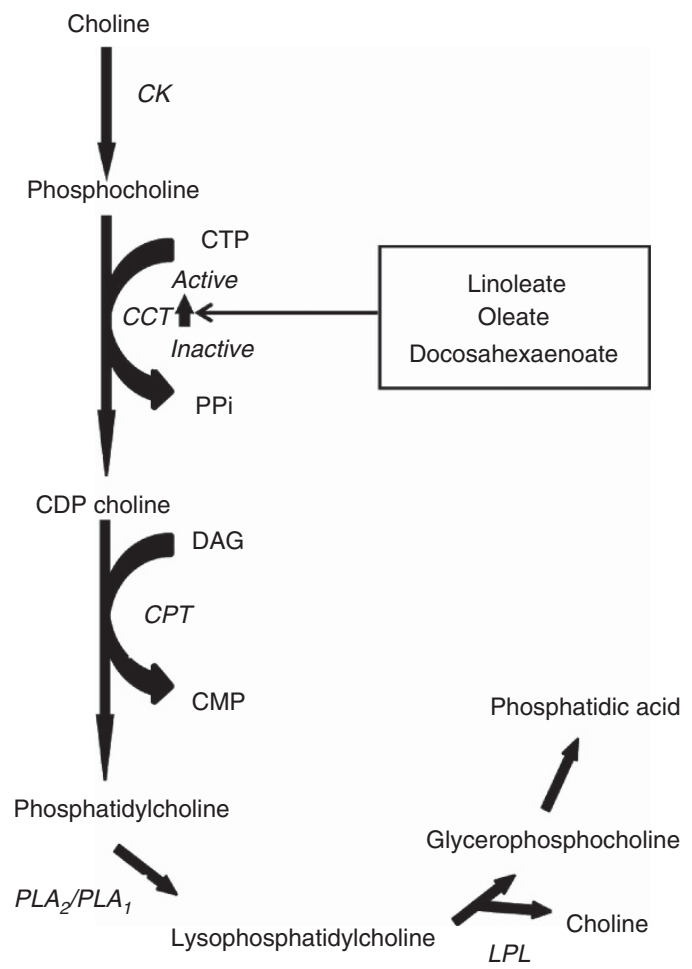

Figure 1. The Kennedy pathway of phosphatidylcholine synthesis and breakdown to phosphatidic acid. PLA $2 / A_{1}$, phospholipase $A_{2} / A_{1} ; L P L$, lysophospholipase. (Kennedy and Weiss, 1956; Fagone and Jackowski, 2013). different models of pancreatic cancer, we were able to probe the Kennedy pathway for correlants of the observed GdPC/dFdCTP ratios that could be important determinants of $\mathrm{dFdC}$ pharmacokinetics and pharmacodynamics.

\section{MATERIALS AND METHODS}

Mouse studies. All mouse experiments were carried out in accordance with the UK Animals (Scientific Procedures) Act 1986, revised by the Amendment Regulations 2012 (SI 2012/3039) to transpose European Directive 2010/63/EU, with approval from the local Animal Welfare and Ethical Review Body, and following the 2010 guidelines from the United Kingdom Coordinating Committee on Cancer Research (Workman et al, 2010).

KPC mouse model of PDAC has been described previously (Hingorani et al, 2005; Olive et al, 2009). KPC mice develop advanced PDAC from 2 to 3 months and have a shortened median survival of approximately 5 months.

Enrolment of KPC mice in the study was based on tumour size, measured by ultrasound in an axial orientation using the Vevo 770 System (Visual Sonics, Inc., Toronto, ON, Canada) (Cook et al, 2008; Olive et al, 2009). Mice with mean PDAC tumour diameters of $6-9 \mathrm{~mm}$ were enrolled.

K8484 is a cell line that was established from a KPC PDAC tumour as described previously (Olive et al, 2009). Control littermates of KPC mice, $\mathrm{p} 53^{\mathrm{R} 172 \mathrm{H}}$; Pdx1-Cre (PC) female mice were used in this study to transplant $1 \times 10^{6} \mathrm{~K} 8484$ cells in PBS subcutaneously into each flank. The mice were dosed with $\mathrm{dFdC}$ when the tumours reached at least $200 \mathrm{~mm}^{3}$. Three mice were used per time point (two tumours per mouse).

Subcutaneous xenografts of MIA PaCa-2 cells were established by implanting $5 \times 10^{6}$ cells in the flank of female 9-week-old $\mathrm{BALB} / \mathrm{c}$ nude mice (Charles River UK Ltd., Margate, UK). The mice were dosed with $\mathrm{dFdC}$ when the tumours reached at least $300 \mathrm{~mm}^{3}$. At least three mice were used per time point.

$\mathrm{dFdC} \mathrm{HCl}$ (Tocris Bioscience, Bristol, UK) was dissolved in sterile $0.9 \%$ saline to $20 \mathrm{mg} \mathrm{ml}^{-1}$ and then used to dose the mice. For PK studies, the mice were killed at fixed time points after a single dose of $100 \mathrm{mg} \mathrm{kg}^{-1} \mathrm{IP} \mathrm{dFdC}$, and plasma and tumour were then snap-frozen. For efficacy studies, mice were dosed twice per week with $100 \mathrm{mg} \mathrm{kg}^{-1} \mathrm{IP} \mathrm{dFdC}$, as used in previous therapeutic studies (Frese et al, 2012; Courtin et al, 2013).

Identification of GdPC. To identify GdPC, an LC-MS/MS method for the physiological metabolite CDP choline (C0256, Sigma Aldrich Co Ltd., Dorset, UK) based on the method by (Desoubzdanne et al, 2010) was developed. A Sciex 4000 Q trap mass spectrometer (AB Sciex UK Ltd., Warrington, UK) fitted with a Turbo ionspray source at $500{ }^{\circ} \mathrm{C}$ operated in positive mode was used. Quantitative data acquisition was done using the software Analyst ver 1.4.2 (Ab Sciex UK Ltd.). Given the similarities in structure between CDP choline and GdPC (Figure 2A and B), their chromatographic properties were also expected to be similar. PDAC tissue, obtained from a mouse $2 \mathrm{~h}$ after IP injection of $\mathrm{dFdC}$ $\left(100 \mathrm{mg} \mathrm{kg}^{-1}\right)$, was precipitated using $100 \%$ acetonitrile. After centrifugation, the supernatant was transferred to a clean tube and evaporated to dryness. The residue was reconstituted in $75 \%$ acetonitrile and injected into the mass spectrometer. An enhanced product ion scan for the expected precursor ion for GdPC $(\mathrm{m} / \mathrm{z}$ 509) was run (under the same conditions as for the multiple reaction monitoring experiment for CDP choline). The spectra obtained were compared with those of CDP choline to aid identification of GdPC.

Quantitation of dFdC, dFdU, dFdCTP, GdPC, Cytidine, CTP and CDP choline by LC-MS/MS in tumour tissue and cell extracts. GdPC (Cat. no. G305015) was synthesised by Toronto 
A

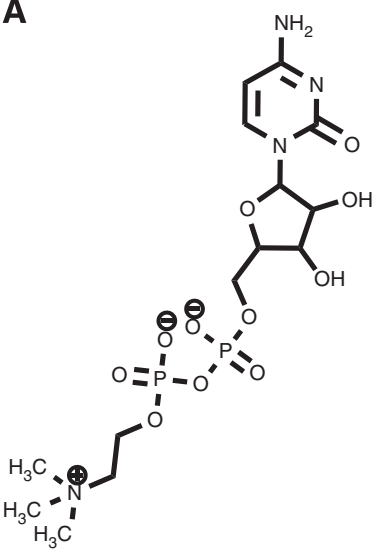

C

C.2e5

$2.1 \mathrm{e} 5$

$2.0 \mathrm{e} 5$
$1.9 \mathrm{e} 5$

$1.8 \mathrm{e}$

1.6

$1.4 \mathrm{e}$

응 $1.3 e 5$

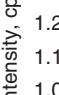

焉 $1.0 \mathrm{e} 5$
$9.0 \mathrm{e} 4$

$9.0 \mathrm{e} 4$
$8.0 \mathrm{e} 4$

$7.0 \mathrm{e} 4$

$6.0 \mathrm{e} 4$

$5.0 \mathrm{e}$
$4.0 \mathrm{e}$
$3.0 \mathrm{e}$

$3.0 \mathrm{e} 4$

$2.0 \mathrm{e} 4$

$1.0 \mathrm{e} 4-112.1136 .1$

$0.0429 .1 \quad 184$

$\begin{array}{lllllll}100 & 120 & 140 & 160 & 180 & 200 & 220\end{array}$

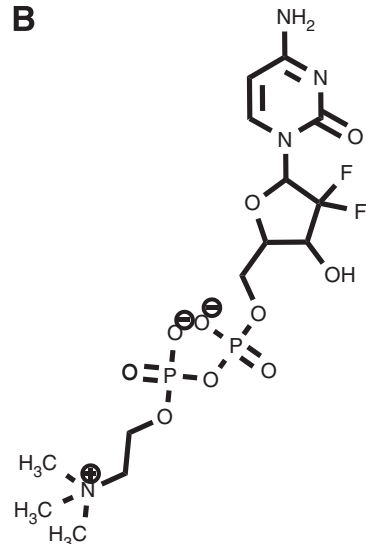

Figure 2. The identification of GdPC by LC-MS/MS. Chemical structures of (A) the physiological metabolite CDP choline, (B) GdPC and (C) enhanced product ion scan spectrum of GdPC (precursor, at $\mathrm{m} / \mathrm{z} 509$ ) and proposed fragmentation.

Research Chemicals (Toronto, ON, Canada) while CTP and cytidine were purchased from Sigma Aldrich Co Ltd. The analytes in tumour tissues were quantified essentially as previously described (Bapiro et al, 2011). Minor modifications included addition of MRM transitions for GdPC (509-184.05), CTP (484-111.91), cytidine (244-112.04) and CDP choline (489.1-184.05). In addition, the mobile phase gradient starting conditions were modified to $98 \%$ ammonium acetate $\mathrm{pH} 10$ and $2 \%$ acetonitrile instead of $95 \%$ and $5 \%$, respectively. To obtain cell extracts from culture in vitro, cells were harvested by trypsinisation and counted. The cells were then washed with ice-cold PBS, and pellets were obtained by centrifugation at $20000 \mathrm{~g}$ for $20 \mathrm{~min}$. Ice-cold $60 \%$ methanol (volume adjusted for number of cells) containing tetrahydrouridine $\left(25 \mu \mathrm{g} \mathrm{ml}^{-1}\right)$ was added to cell pellets and vortexed vigorously. The cell methanol mixture was incubated at $-80^{\circ} \mathrm{C}$ for at least $30 \mathrm{~min}$ and then sonicated for $10 \mathrm{~min}$ in an ice-bath. In all, $50 \mu$ l of the cell extract was then processed as described above for the tumour tissue. Absolute concentrations were obtained for $\mathrm{dFdC}, \mathrm{dFdU}, \mathrm{dFdCTP}$ and GdPC, because these are non-endogenous metabolites, and standard curves could be created using non-dFdC-treated tissue or cell homogenates as the blank matrix. CDP choline, CTP and cytidine were expressed as relative concentrations (based on MS peak area ratio of analyte $v s$ internal standard), because these are endogenous metabolites, and so no blank matrix was obtainable to use for standard curves.

Quantitation of phosphocholine, glycerophosphocholine and total choline containing metabolites by NMR. In all, $25 \mathrm{mg}$ of tumour tissue from untreated mice and from mice $4 \mathrm{~h}$ post-dFdC $\left(100 \mathrm{mg} \mathrm{kg}^{-1}\right)$ IP were homogenised in $50 \%$ acetonitrile using a Precellys homogeniser (Stretton Scientific, Stretton, UK) in reinforced tubes with small ball bearings (Precellys MK28-R) for $2 \times 50 \mathrm{~s}$ at $5800 \mathrm{r}$.p.m. After centrifugation at $20000 \mathrm{~g}$ for $20 \mathrm{~min}$, the supernatant was transferred to a clean tube and evaporated to dryness. The residue was dissolved in $0.5 \mathrm{ml}$ of $\mathrm{D}_{2} \mathrm{O}$ and transferred to a $5 \mathrm{~mm}$ standard NMR tube; $10 \mu \mathrm{l}$ of $10 \mathrm{~mm}$ DSS (4,4-dimethyl-4-silapentane-1-sulphonic acid) was then added as an external standard. The NMR tubes were then loaded onto a $600 \mathrm{MHz}$ Bruker Avance NMR spectrometer (Bruker UK Ltd., Coventry, UK). NMR data was then acquired with a water presaturation pulse sequence. ${ }^{1} \mathrm{H}$ NMR spectroscopic acquisition parameters included $32 \mathrm{k}$ time domain data points, 512 averages, repetition delay of $5 \mathrm{~s}$ and acquisition time of $2.2 \mathrm{~s}$, resulting in a total acquisition time of $1 \mathrm{~h} 2 \mathrm{~min}$ for each spectrum. The acquired data were Fourier transformed, phase corrected and the baseline was corrected. We used the Bruker TOPSPIN 2.1 software for 
NMR spectral acquisition and processing and the NMR Suite 7.5 (Chenomx Inc., Edmonton, AB, Canada) software package for identification and quantitation of metabolites. The DSS signal was used to identify the chemical shifts of metabolites and to estimate the absolute metabolite concentrations.

Cell culture. The K8484 cell line was maintained in DMEM (D6429, Sigma Aldrich Co Ltd.) containing 5\% FBS (10270-098, Life Technologies Ltd., Paisley, UK). MIA PaCa-2 cells were obtained from the European Collection of Cell Cultures (Public Health England, Salisbury, UK, authenticated by STR genotyping) and maintained in DMEM containing 10\% FBS. Gemcitabine (3259, Tocris Bioscience) was dissolved in DMSO; linoleate (L8134, Sigma Aldrich Co Ltd.) and 6-diazo-5-oxo-L-norleucine (DON, D2141 Sigma Aldrich Co Ltd.) were dissolved in sterile water. Experiments assessing the effect of DON on $\mathrm{dFdC}$ metabolism were carried out in media lacking glutamine (D5671, Sigma Aldrich Co Ltd.). Growth-inhibition assays were carried out using the sulphorhodamine assay, and subsequent synergy calculations were done as previously described (Lin et al, 2012).

Effect of CTP on GdPC formation in tumour homogenate. KPC PDAC tissue homogenised in $0.1 \mathrm{M}$ Tris-Cl buffer $\mathrm{pH} 7.4$ (1:4) was used as the source of CCT enzyme. The reaction mixture in a final volume of $200 \mu \mathrm{l}$ consisted of tissue homogenate $(10 \mu \mathrm{l})$, magnesium chloride $(8 \mathrm{~mm})$, EDTA $(1.8 \mathrm{~mm})$, phosphocholine $(1 \mathrm{mM})$ and Tris-Cl buffer $\mathrm{pH} 7.4(0.1 \mathrm{M})$. The reaction was started by adding dFdCTP $(200 \mu \mathrm{M})$ and incubated at $37^{\circ} \mathrm{C}$ for $30 \mathrm{~min}$, and the reaction was stopped by adding $200 \mu \mathrm{l}$ of ice-cold acetonitrile (100\%). The effect of CTP $(200 \mu \mathrm{m})$ on GdPC formation was assessed by adding CTP to the reaction mixture. A control sample was incubated under the same conditions, with dFdCTP added after stopping the reaction. After centrifugation at $20000 \mathrm{~g}$ for $20 \mathrm{~min}$, the supernatant was transferred to a clean tube and evaporated to dryness. The residue was reconstituted in $100 \mu \mathrm{l}$ water, and $15 \mu \mathrm{l}$ was used for quantitation of GdPC using the LC-MS/MS method mentioned above.
Statistical analysis. Statistical analyses were performed using the GraphPad Prism version 6 (GraphPad Software Inc., La Jolla, CA, USA). A one-way ANOVA was used followed by Holm-Sidak's multiple comparison posttest.

\section{RESULTS}

Identification and quantitation of GdPC. LC-MS/MS analysis was performed on homogenate from a KPC tumour taken $2 \mathrm{~h}$ after a single dose of $\mathrm{dFdC}$. An enhanced product ion spectrum for GdPC of the expected precursor ion $\mathrm{m} / z 509$ yielded spectra adequate for a definitive identification, which was aided by comparison with that of CDP choline $\mathrm{m} / \mathrm{z}$ 489.2, given their similar structures (Figure 2A-C). The fragment ions at $\mathrm{m} / z$ 184.1, 166 and 125 are characteristic of the phosphocholine moiety (Vaezian et al, 2010; Lattanzio et al, 2009; Hayashi et al, 1989; Antonopoulou et al, 2002). A sample of GdPC was then customsynthesised for use in standard curves for subsequent quantification of GdPC in tumour homogenates.

PDAC models. In order to investigate the significance of GdPC, we next assessed concentrations of dFdCTP and GdPC in in vivo models of pancreatic cancer. dFdC shows little efficacy in KPC mouse PDAC tumours (Olive et al, 2009). The intra-tumoural concentrations of $\mathrm{dFdC}, \mathrm{dFdU}, \mathrm{dFdCTP}$ and GdPC were measured in KPC mice after administration of $100 \mathrm{mg} \mathrm{kg}^{-1} \mathrm{dFdC}$, and samples were collected at $1,2,4,6$ and $10 \mathrm{~h}$ with at least five animals per time point. GdPC and dFdCTP exhibited similar concentration time profiles in KPC mice, and maximum concentrations were observed at about $2 \mathrm{~h}$ (Figure $3 \mathrm{~A}$ ). Peak dFdCTP was $6.1 \pm 3.9$ pmoles $^{-1} \mathrm{mg}^{-1}$ tissue and remained detectable at $10 \mathrm{~h}$ in only one of the samples. Peak GdPC was $7.2 \pm 3.8$ pmoles $\mathrm{mg}^{-1}$ at $2 \mathrm{~h}$ and below the level of quantification by $10 \mathrm{~h}$. The concentration time profile for $\mathrm{dFdC}$ and $\mathrm{dFdU}$ in the same samples is shown in Supplementary Figure S1A. We then investigated whether GdPC could be detected in other in vivo
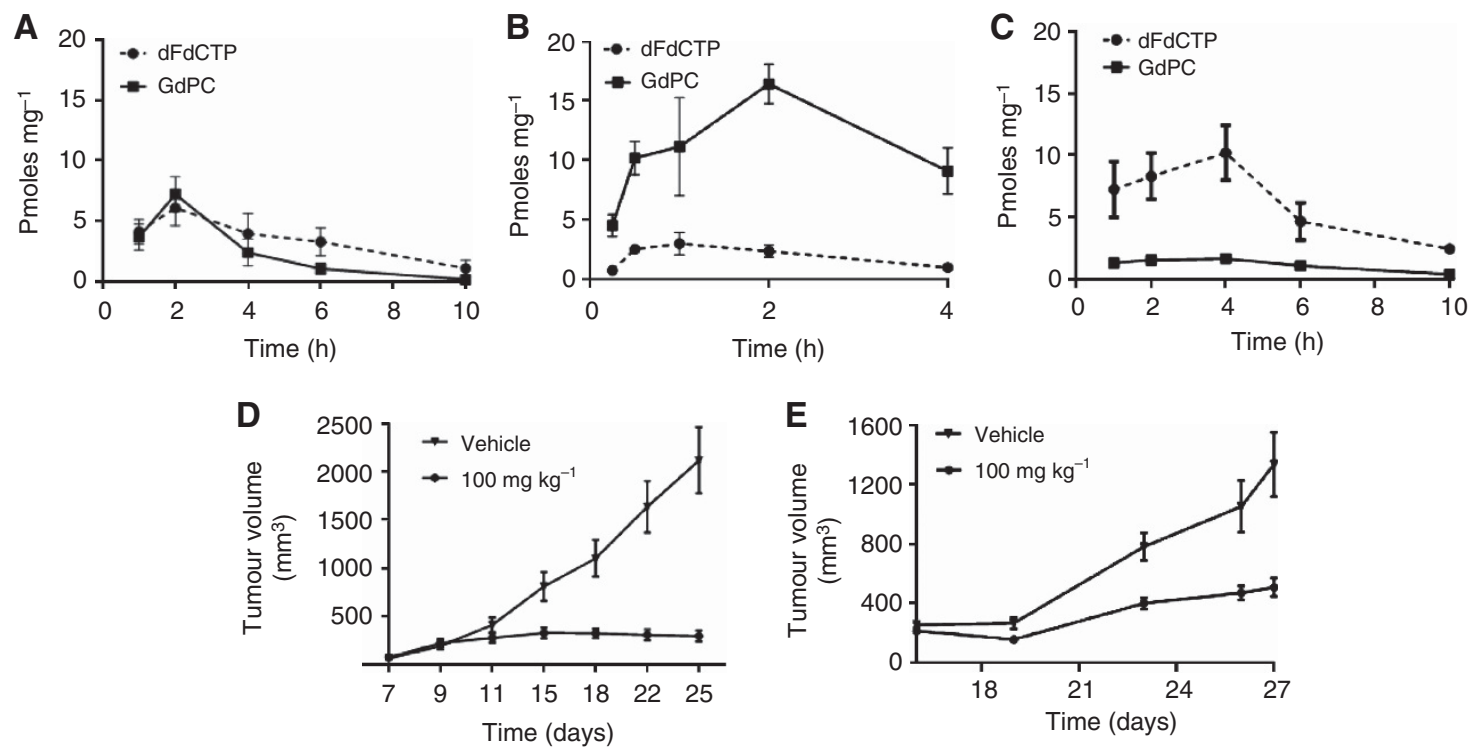

Figure 3. $\mathrm{dFdC}$ metabolism and efficacy in PDAC models in vivo. Tumour GdPC and dFdCTP concentrations after IP administration of dFdC $\left(100 \mathrm{mg} \mathrm{kg}^{-1}\right)$ with results expressed as mean \pm s.e.m. in (A) KPC mice, with seven mice per time point except 10-h time point where five mice were used. We have recently shown the dFdCTP concentration time profile (Neesse et al, 2013). (B) In K8484 (KPC PDAC cell) allograft-bearing mice, both analytes were below the limit of quantitation (BLQ) at the 24-h time point with three mice per time point except at the 1-h time point where $n=2$; and (C) in MIA PaCa-2 xenograft-bearing mice, GdPC was BLQ at the 24-h time point with at least three mice per time point (results are the mean \pm s.e.m.). (D) The effect of $\mathrm{dFdC}$ on tumour volumes (mean \pm s.e.m.) with time in days after tumour implantation in K8484 allografts, which were dosed on days $8,11,15,18,22$ and 25 ( $n=10$ per group), and (E) tumour volumes in MIA PaCa-2 xenografts dosed with $\mathrm{dFdC}$ on days $16,19,23$ and 26 ( $n=10$ per group). 
pancreatic cancer models used in our laboratory - first, in subcutaneous allografts of a cell line K8484 derived from a KPC PDAC tumour. This allograft model responds very well to $\mathrm{dFdC}$ (Figure 3D). For PK analysis, K8484 cells were transplanted subcutaneously into syngeneic $\mathrm{Kras}^{\mathrm{wt}}$; p53 ${ }^{\mathrm{R} 172 \mathrm{H}}$; Pdx1-Cre (PC) mice, and once the tumours had grown sufficiently, a single dose of $\mathrm{dFdC}$ (100 $\mathrm{mg} \mathrm{kg}^{-1}$, IP) was administered. Tumour tissue samples were collected at $0.25,0.5,1,2,4$ and $24 \mathrm{~h}$ and were used to quantify $\mathrm{dFdC}$ and metabolites. To our surprise and in contrast to results in the KPC mouse tumour tissue, GdPC was the predominant metabolite, with at least a four-fold higher AUC than dFdCTP (Figure 3B). The peaks were maximal at 1 and $2 \mathrm{~h}$ for dFdCTP and GdPC, respectively, and both metabolites were below the limit of quantification at $24 \mathrm{~h}$. Of note, the absolute dFdCTP concentrations in the K8484 allograft (peak $3.0 \pm 2.0$ pmoles $\mathrm{mg}^{-1}$ ) were lower than those in the KPC model, but the

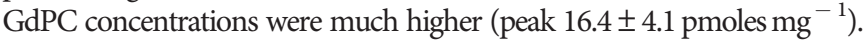
The concentration time profile for $\mathrm{dFdC}$ and $\mathrm{dFdU}$ in the same tumours is shown in Supplementary Figure S1B.

While mindful of the complexities of comparing different species, we have also assessed amounts of dFdCTP and GdPC in the MIA Paca-2 xenograft, another model that is sensitive to growth inhibition by dFdC (Figure 3E). In MIA PaCa-2 xenograft PK studies, after a single dose of $\mathrm{dFdC}$, dFdCTP was the predominant metabolite over GdPC (Figure 3C), the opposite result to that seen in K8484 allografts. The peak concentration of $\mathrm{dFdCTP}$, at $4 \mathrm{~h}$, was $10.2 \pm 5.0$ pmoles $\mathrm{mg}^{-1}$ and that of GdPC was

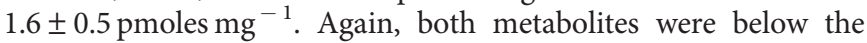
limit of quantification at $24 \mathrm{~h}$. The concentration time profile for $\mathrm{dFdC}$ and $\mathrm{dFdU}$ in the same tumours is shown in Supplementary Figure S1C. Representative H\&E images of tumour sections from each of the three models are shown in Supplementary Figure S2.
We next investigated whether differences in Kennedy pathway intermediates could explain the differences in amounts of dFdCTP and GdPC observed in the different in vivo models of pancreatic cancer.

Comparison of Kennedy pathway intermediates in tumour tissue from in vivo PDAC models. ${ }^{1} \mathrm{H}$ HR NMR spectroscopy was used to measure concentrations of Kennedy pathway intermediates phosphocholine, glycerophosphocholine and total choline-containing metabolites in tumour homogenates from KPC PDAC, K8484 allografts and MIA PaCa-2 xenografts taken before and $4 \mathrm{~h}$ after IP administration of $\mathrm{dFdC}\left(100 \mathrm{mg} \mathrm{kg}^{-1}\right)$ (Supplementary Figure S3). No differences were observed in the concentrations of the Kennedy pathway metabolites between samples from dFdC-dosed and untreated mice in each group. The only significant difference was in the phosphocholine/ glycerophosphocholine ratio, when $\mathrm{dFdC}$-dosed K8484 allografts were compared with $\mathrm{dFdC}$-dosed MIA Paca-2 xenografts (Supplementary Figure S3C). Using our LC-MS/MS assay, we then quantified relative levels of CDP choline and CTP in tumour homogenates of mice from the three different models taken $2 \mathrm{~h}$ after administration of dFdC. Although no significant difference was observed in the relative concentrations of CDP choline (Figure 4A), both CTP and cytidine exhibited an inverse correlation with the concentration of GdPC (Figure 4B and D). The K8484 allografts had the highest concentrations of GdPC and the lowest concentrations of CTP, while MIA PaCa-2 xenografts had the lowest GdPC but highest CTP concentration. We hypothesised that the inverse correlation between CTP and GdPC might be caused by inhibition of GdPC formation by CTP. Therefore an in vitro assay was established to measure formation of GdPC using a
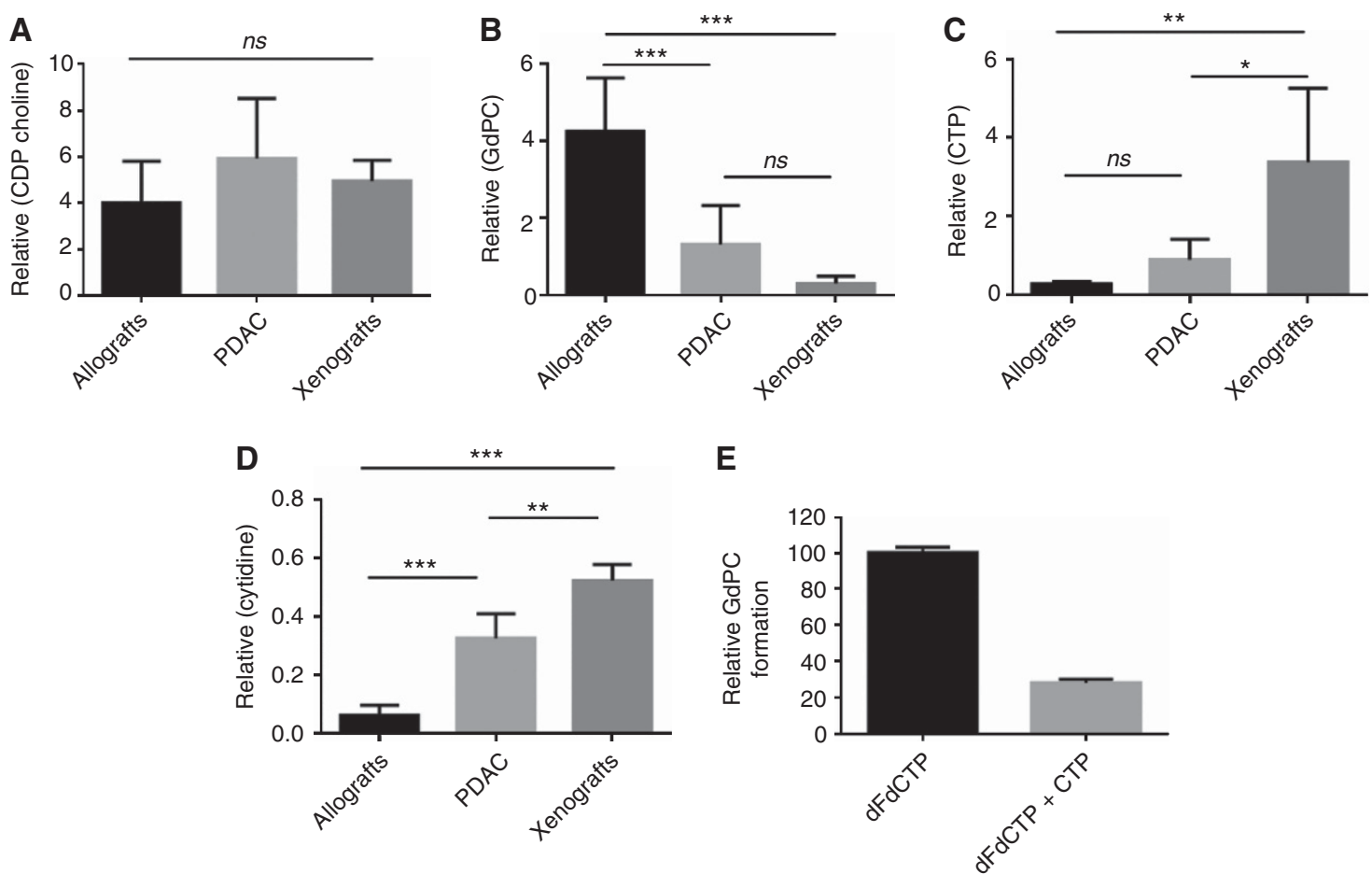

Figure 4. Relative analyte concentrations $2 \mathrm{~h}$ post-IP dose of $100 \mathrm{mg} \mathrm{kg}^{-1} \mathrm{dFdC}$. 'Relative concentrations' are peak area ratios of analyte vs internal standard. (A) CDP choline in KPC PDAC $(n=5)$, K8484 allografts $(n=4)$ and MIA PaCa-2 xenografts $(n=6),(B)$ GdPC in PDAC $(n=7)$, K8484 allografts $(n=3)$ and MIA PaCa-2 xenografts $(n=2)$, (C) CTP in K8484 allografts $(n=4)$, MIA PaCa-2 xenografts $(n=6)$ and KPC PDAC $(n=5)$, (D) cytidine in tumour tissue $(n=4)$ for all of K8484 allografts, MIA PaCa-2 xenografts and KPC PDAC (NS, not significant $P>0.05$; $P \leqslant 0.05$; $\left.{ }^{\star \star} P \leqslant 0.01 ;{ }^{* \star \star} P \leqslant 0.001 ;{ }^{* \star \star \star} P \leqslant 0.0001\right)$. (E) Effect of CTP $(200 \mu \mathrm{m})$ on formation of GdPC by PDAC homogenate incubated with dFdCTP (200 $\left.\mu \mathrm{m}\right)$ in vitro. Results are mean \pm s.d. of triplicate samples. 
homogenate of KPC PDAC tissue as an enzyme source and dFdCTP as the substrate, and this assay was used to assess the effect of CTP. CTP was found to be an inhibitor of GdPC formation (Figure 4E). Treating K8484 cells in vitro with the CTP synthase inhibitor DON resulted in $>50$-fold reduction in CTP levels, accompanied by a 15 -fold increase in the GdPC concentration (Supplementary Figure S4), confirming that CTP inhibits GdPC formation.

Investigation of whether GdPC contributes to $\mathrm{dFdC}$ anti-tumour activity. Comparison of the GdPC/dFdCTP profiles in the three in vivo models (Figure 3 ) with the relative efficacy of $\mathrm{dFdC}$ in those models suggests a role for GdPC in $\mathrm{dFdC}$ antitumour activity assessed on the basis of effect on tumour volume. After adding $\mathrm{dFdC}(100 \mathrm{nM})$ to $\mathrm{K} 8484$ cells in vitro, in sharp contrast to the results with K8484 allografts in vivo, it was apparent that $\mathrm{dFdCTP}$ (Figure 5A) was the predominant metabolite rather than GdPC (Figure 5B), and in other PDAC cell lines incubated with $\mathrm{dFdC}$ the in vitro GdPC concentration was rarely above the lower limit of quantification (data not shown). Attempts to assess the cytotoxicity of GdPC by adding increasing concentrations of the compound to cells in culture failed, because it was degraded to $\mathrm{dFdC}$ in the culture medium (even in the absence of cells) - the highest concentration used, $300 \mathrm{~nm}$ of GdPC, was almost completely converted to $\mathrm{dFdC}$ within $24 \mathrm{~h}$ of incubation at $37^{\circ} \mathrm{C}$ (data not shown). Thus, manipulation of the intracellular generation of GdPC was felt to be the only feasible approach to assess its impact on cytotoxicity. Linoleic acid has been shown to activate CCT and synthesis of PtdCho (Mallampalli et al, 1994; Burkhardt et al, 1988), so we assessed the effects of adding linoleic acid on the cytotoxic effects of $\mathrm{dFdC}$ in K8484 cells using an $8 \times 8$ concentration combination assay, incubating for $72 \mathrm{~h}$. We observed significant synergistic effects with $E_{\max }$ of $99 \%$ growth inhibition at $10 \mathrm{~nm} \mathrm{dFdC}$ and $170 \mu \mathrm{m}$ linoleate, compared with a prediction of $57 \%$ growth inhibition if the effect of the two drugs were additive in accordance with the Bliss Additivity model (Figure 5C). Shorter incubations with $170 \mu \mathrm{m}$ linoleate were used to assess its effect on dFdC (100 nM) metabolism: dFdCTP concentrations were unaffected by linoleate (Figure 5A), but there was a 10 -fold increase in GdPC concentration at $2.5 \mathrm{~h}$, and it continued to be higher over $10 \mathrm{~h}$ (Figure 5B). Thus linoleate increased the cellular GdPC concentration after $\mathrm{dFdC}$ administration and enhanced $\mathrm{dFdC}$ cytotoxicity synergistically.

\section{DISCUSSION}

For the past two decades, $\mathrm{dFdC}$ alone or in combination has been the standard of care for PDAC (reviewed in Michl and Gress, 2013), with few new therapeutic advances resulting in improved survival. One exception is FOLFIRINOX, a combination devoid of $\mathrm{dFdC}$ which recently showed increased survival in PDAC (Conroy et al, 2011) but is too toxic to be used routinely in the majority of patients. dFdC combinations continue to be important, as exemplified by results of the recently completed phase III trial of a nab-paclitaxel/dFdC combination (Von Hoff et al, 2013). Part of the mechanism of efficacy of this combination may be the ability of nab-paclitaxel to enhance $\mathrm{dFdC}$ efficacy via modulation of its metabolism by cytidine deaminase (Frese et al, 2012). We have now used the KPC model to shed new light on the intra-tumoural metabolism of $\mathrm{dFdC}$ with the hope of uncovering novel metabolic targets with potential to improve the drug's efficacy. We have
A

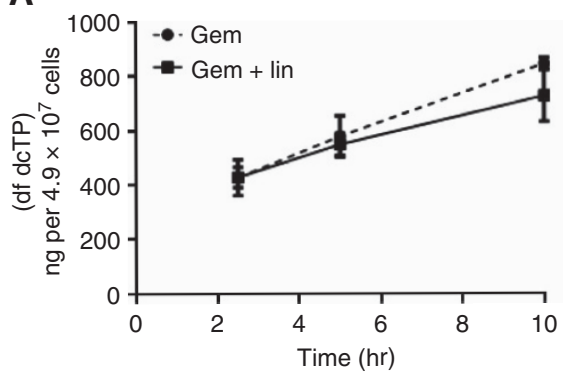

B

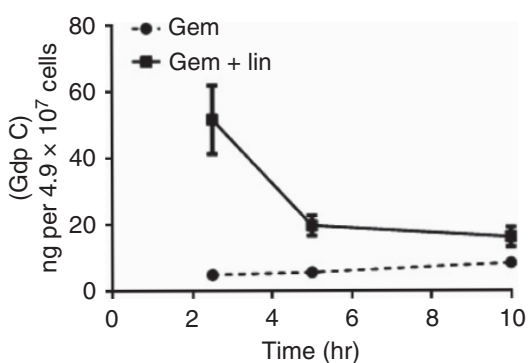

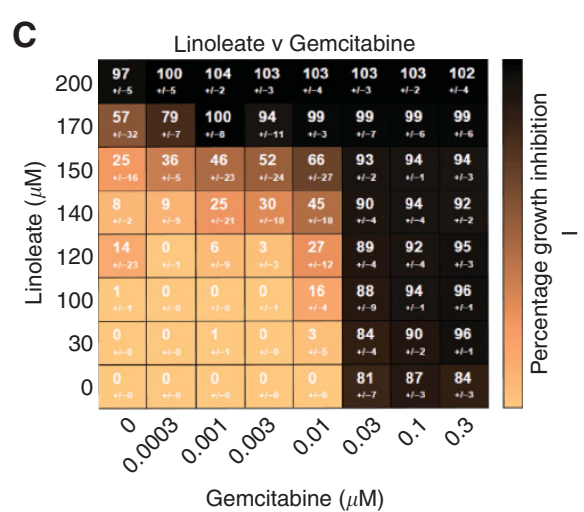

Gemcitabine $(\mu \mathrm{M})$

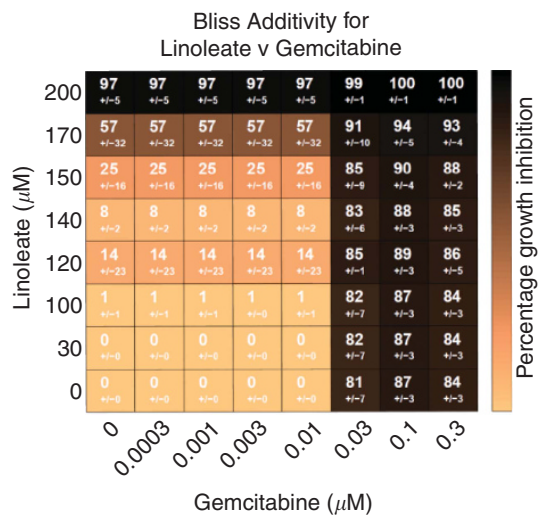

Gemcitabine $(\mu \mathrm{M})$
Data - Bliss additivity for Linoleate $v$ Gemcitabine

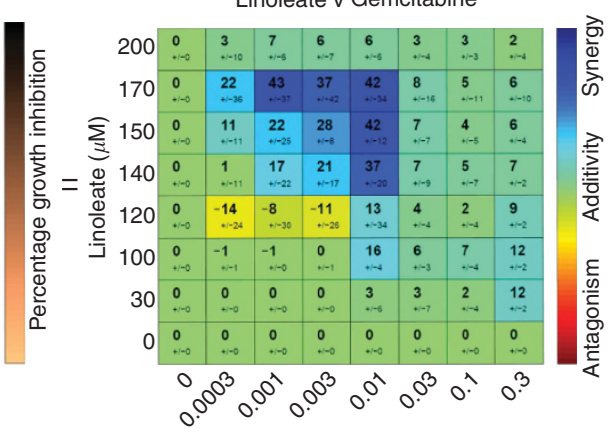

Gemcitabine $(\mu \mathrm{M})$

Figure 5. The effect of the combination of linoleate and gemcitabine in K8484 cells. The timecourse of production of (A) dFdCTP and (B) GdPC in K8484 cells grown in DMEM supplemented with $5 \% \mathrm{FBS}$ and treated over $10 \mathrm{~h}$ with linoleate $(170 \mu \mathrm{M})$ and dFdC (100 nM). Results are the mean \pm s.d. of three replicates. (C) Effect on growth of K8484 cells using the sulphorhodamine B cytotoxicity assay, exposed to an $8 \times 8$ combination of linoleate and dFdC for $72 \mathrm{~h}$. The left panel shows the experimental data (mean of three independent experiments, each done in triplicate) and shows the percentage of growth inhibition compared with DMSO control. A Bliss additivity model built from the experimental single agent data shows the predicted growth inhibition if the combination were additive (middle). The right panel shows the difference values after subtracting the predicted values from the experimental data, with difference values of $>25$ considered to show synergy (in blue). 


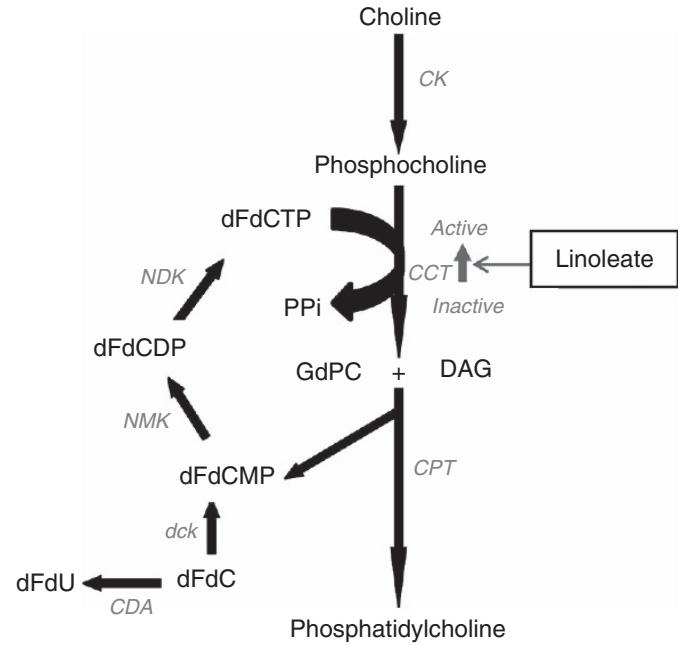

Figure 6. The proposed point at which $\mathrm{dFdC}$ metabolism interacts with the Kennedy pathway that converts choline to phosphatidylcholine, resulting in formation of GdPC, and the impact of linoleate on that reaction.

shown here that the Kennedy pathway-linked GdPC is a major metabolite of $\mathrm{dFdC}$ in vivo. The metabolic pathway by which it is formed is shown in Figure 6.

Compounds analogous to this CDP choline metabolite have been shown to be formed from compounds with similar structures to $\mathrm{dFdC}$, including 2',3'-dideoxycytidine (zalcitabine), zebularine and arabinofuranosylcytosine (ara-C) (Lauzon et al, 1978a,b; Rossi et al, 1999; Ben-Kasus et al, 2005). An unidentified metabolite $\mathrm{X}$, making up $2.8 \%$ of total $\mathrm{dFdC}$, was reported in Chinese Hamster Ovary cells in studies using radiolabeled dFdC (Heinemann et al, 1988), which they suggested could be the 'CDP choline analogue similar to that found in cells treated with ara-C'. As shown by our in vitro studies, in cultured cells, dFdCTP is the predominant metabolite rather than GdPC, with GdPC concentrations rarely above the limit of quantification, which explains why $\mathrm{dFdC}$ metabolism experiments conducted in cultured cells would probably fail to detect GdPC as an important metabolite.

The three in vivo PDAC models showed different intratumoural GdPC/dFdCTP ratios that inversely correlated with CTP concentrations, a feature that was explained by the inhibition of GdPC formation by CTP observed in vitro using tumour homogenates. It is, however, not clear why there are differences in the CTP concentrations. An important difference of the models used relates to their stromal component. The fact that we are able to detect GdPC in both subcutaneous xenografts (which have little stroma) and the authochthonous PDAC in KPC mice (with extensive stroma) suggests that its production is not specific to tumour stroma.

The greater efficacy of $\mathrm{dFdC}$ in the K8484 allografts and the predominance of GDPC over dFdCTP in this model implicated GdPC as a possible cytotoxic metabolite, so that mechanism was investigated in cells in vitro.

Ideally, cytotoxic effects of GdPC would have been easily studied by adding increasing concentrations to cultured cells; however, this was not possible because of the instability of GdPC in culture medium, coupled with its chemical structure (charged choline and phosphate groups at physiological $\mathrm{pH}$ ) which is likely to prevent it from crossing the plasma membrane. Generating the metabolite intracellularly offered a solution to this problem, and this was achieved by combining $\mathrm{dFdC}$ with linoleic acid, which is known to activate CCT, resulting in synergistic cytotoxicity. The mechanism of the observed synergistic cytotoxicity accompanying the increased GdPC concentration is not clear. The equivalent metabolite of ara-C at very high concentrations $(400 \mu \mathrm{M})$ is thought to cause excessive formation of PtdCho resulting in cell lysis (Daly et al, 1990) but is unlikely to be the case in our study because a 1000 -fold lower concentration of $\mathrm{dFdC}$ was used. The clinical relevance of our findings is supported by reports of an on-going clinical trial suggesting better outcomes in pancreatic cancer patients treated with $\mathrm{dFdC}$ and the fish oil formulation Lipidem (B. Braun Melsungen AG, Melsungen, Germany), one of whose major constituents is linoleic acid (Arshad et al, 2013a,b). We believe that Lipidem may promote formation of the active form of CCT and might also increase tumoural concentrations of GdPC.

In conclusion, we have established GdPC as an important element of the $\mathrm{dFdC}$ metabolic pathway in tumour tissue in mouse models of PDAC and shown that its formation may contribute to dFdC cytotoxicity.

\section{ACKNOWLEDGEMENTS}

We thank the CRUK Cambridge Institute BRU staff for assistance with mouse studies and Donna-Michelle Smith and Michael Williams for advice on LC-MS/MS. TEB, AC, JLB and FMR were funded by Cancer Research UK programme grant C96/A8333 Senior Group Leader Funding to DIJ. KKF, NC and AN were funded by Cancer Research UK Senior Group Leader Funding to DAT. BM was funded by Cancer Research UK Senior Group Leader Funding to JRG.

\section{CONFLICT OF INTEREST}

The authors declare no conflict of interest.

REFERENCES

Antonopoulou S, Oikonomou A, Karantonis HC, Fragopoulou E, Pantazidou A (2002) Isolation and structural elucidation of biologically active phospholipids from Scytonema julianum (cyanobacteria). Biochem J 367(Pt 1): 287-293.

Arshad A, Chung W, Isherwood J, Steward W, Metcalfe M, Dennison A (2013a) Restoration of mannose-binding lectin complement activity is associated with improved outcome in patients with advanced pancreatic cancer treated with gemcitabine and intravenous omega-3 fish oil. JPEN J Parenter Enteral Nutr 38(2): 214-219.

Arshad A, Chung WY, Steward W, Metcalfe MS, Dennison AR (2013b) Reduction in circulating pro-angiogenic and pro-inflammatory factors is related to improved outcomes in patients with advanced pancreatic cancer treated with gemcitabine and intravenous omega-3 fish oil. HPB (Oxford) 15(6): 428-432.

Bapiro TE, Richards FM, Goldgraben MA, Olive KP, Madhu B, Frese KK, Cook N, Jacobetz MA, Smith DM, Tuveson DA, Griffiths JR, Jodrell DI (2011) A novel method for quantification of gemcitabine and its metabolites $2^{\prime}, 2^{\prime}$-difluorodeoxyuridine and gemcitabine triphosphate in tumour tissue by LC-MS/MS: comparison with (19)F NMR spectroscopy. Cancer Chemother Pharmacol 68(5): 1243-1253.

Ben-Kasus T, Ben-Zvi Z, Marquez VE, Kelley JA, Agbaria R (2005) Metabolic activation of zebularine, a novel DNA methylation inhibitor, in human bladder carcinoma cells. Biochem Pharmacol 70(1): $121-133$.

Beumer JH, Eiseman JL, Parise RA, Joseph E, Covey JM, Egorin MJ (2008) Modulation of gemcitabine (2',2'-difluoro-2'-deoxycytidine) pharmacokinetics, metabolism, and bioavailability in mice by 3,4,5,6-tetrahydrouridine. Clin Cancer Res 14(11): 3529-3535.

Burkhardt R, Von Wichert P, Batenburg JJ, Van Golde LM (1988) Fatty acids stimulate phosphatidylcholine synthesis and CTP:choline-phosphate cytidylyltransferase in type II pneumocytes isolated from adult rat lung. Biochem J 254(2): 495-500. 
Conroy T, Desseigne F, Ychou M, Bouche O, Guimbaud R, Becouarn Y, Adenis A, Raoul JL, Gourgou-Bourgade S, de la Fouchardiere C, Bennouna J, Bachet JB, Khemissa-Akouz F, Pere-Verge D, Delbaldo C, Assenat E, Chauffert B, Michel P, Montoto-Grillot C, Ducreux M (2011) FOLFIRINOX versus gemcitabine for metastatic pancreatic cancer. $N$ Engl $J$ Med 364(19): 1817-1825.

Cook N, Olive KP, Frese K, Tuveson DA (2008) K-Ras-driven pancreatic cancer mouse model for anticancer inhibitor analyses. Methods Enzymol 439: 73-85.

Costanzi S, Vilar S, Micozzi D, Carpi FM, Ferino G, Vita A, Vincenzetti S (2011) Delineation of the molecular mechanisms of nucleoside recognition by cytidine deaminase through virtual screening. Chem Med Chem 6(8): $1452-1458$.

Courtin A, Richards FM, Bapiro TE, Bramhall JL, Neesse A, Cook N, Krippendorff BF, Tuveson DA, Jodrell DI (2013) Anti-tumour efficacy of capecitabine in a genetically engineered mouse model of pancreatic cancer. PLoS One 8(6): e67330.

Daly PF, Zugmaier G, Sandler D, Carpen M, Myers CE, Cohen JS (1990) Regulation of the cytidine phospholipid pathways in human cancer cells and effects of 1-beta-D-arabinofuranosylcytosine: a noninvasive 31P nuclear magnetic resonance study. Cancer Res 50(3): 552-557.

Desoubzdanne D, Claparols C, Martins-Froment N, Zedde C, Balayssac S, Gilard V, Terce F, Martino R, Malet-Martino M (2010) Analysis of hydrophilic and lipophilic choline compounds in radioresistant and radiosensitive glioblastoma cell lines by HILIC-ESI-MS/MS. Anal Bioanal Chem 398(6): 2723-2730.

Fagone P, Jackowski S (2013) Phosphatidylcholine and the CDP-choline cycle. Biochim Biophys Acta 1831(3): 523-532.

Frese KK, Neesse A, Cook N, Bapiro TE, Lolkema MP, Jodrell DI, Tuveson DA (2012) nab-Paclitaxel potentiates gemcitabine activity by reducing cytidine deaminase levels in a mouse model of pancreatic cancer. Cancer Discov 2(3): 260-269.

Hayashi A, Matsubara T, Morita M, Kinoshita T, Nakamura T (1989) Structural analysis of choline phospholipids by fast atom bombardment mass spectrometry and tandem mass spectrometry. J Biochem 106(2): 264-269.

Heinemann V, Hertel LW, Grindey GB, Plunkett W (1988) Comparison of the cellular pharmacokinetics and toxicity of $2^{\prime}, 2^{\prime}$-difluorodeoxycytidine and 1-beta-D-arabinofuranosylcytosine. Cancer Res 48(14): 4024-4031.

Hingorani SR, Wang L, Multani AS, Combs C, Deramaudt TB, Hruban RH, Rustgi AK, Chang S, Tuveson DA (2005) Trp53R172H and KrasG12D cooperate to promote chromosomal instability and widely metastatic pancreatic ductal adenocarcinoma in mice. Cancer Cell 7(5): 469-483.

Huang P, Chubb S, Hertel LW, Grindey GB, Plunkett W (1991) Action of $2^{\prime}, 2^{\prime}$-difluorodeoxycytidine on DNA synthesis. Cancer Res 51(22): 6110-6117.

Kennedy EP, Weiss SB (1956) The function of cytidine coenzymes in the biosynthesis of phospholipides. J Biol Chem 222(1): 193-214.

Kucera GL, Capizzi RL (1992) 1-Beta-D-arabinofuranosylcytosinediphosphate-choline is formed by the reversal of cholinephosphotransferase and not via cytidylyltransferase. Cancer Res 52(14): 3886-3891.

Lattanzio VM, Baronio M, Oren A, Russell NJ, Corcelli A (2009) Characterization of polar membrane lipids of the extremely halophilic bacterium Salinibacter ruber and possible role of cardiolipin. Biochim Biophys Acta 1791(1): 25-31.

Lauzon GJ, Paran JH, Paterson AR (1978a) Formation of 1-beta-Darabinofuranosylcytosine diphosphate choline in cultured human leukemic RPMI 6410 cells. Cancer Res 38(6): 1723-1729.
Lauzon GJ, Paterson AR, Belch AW (1978b) Formation of 1-beta-Darabinofuranosylcytosine diphosphate choline in neoplastic and normal cells. Cancer Res 38(6): 1730-1733.

Lin Y, Richards FM, Krippendorff BF, Bramhall JL, Harrington JA, Bapiro TE, Robertson A, Zheleva D, Jodrell DI (2012) Paclitaxel and CYC3, an aurora kinase A inhibitor, synergise in pancreatic cancer cells but not bone marrow precursor cells. Br J Cancer 107(10): 1692-1701.

Mallampalli RK, Salome RG, Spector AA (1994) Regulation of CTP:cholinephosphate cytidylyltransferase by polyunsaturated n-3 fatty acids. Am J Physiol 267(6 Pt 1): L641-L648.

Michl P, Gress TM (2013) Current concepts and novel targets in advanced pancreatic cancer. Gut 62(2): 317-326.

Neesse A, Frese KK, Bapiro TE, Nakagawa T, Sternlicht MD, Seeley TW, Pilarsky C, Jodrell DI, Spong SM, Tuveson DA (2013) CTGF antagonism with mAb FG-3019 enhances chemotherapy response without increasing drug delivery in murine ductal pancreas cancer. Proc Natl Acad Sci USA 110(30): 12325-12330.

Olive KP, Jacobetz MA, Davidson CJ, Gopinathan A, McIntyre D, Honess D, Madhu B, Goldgraben MA, Caldwell ME, Allard D, Frese KK, Denicola G, Feig C, Combs C, Winter SP, Ireland-Zecchini H, Reichelt S, Howat WJ, Chang A, Dhara M, Wang L, Ruckert F, Grutzmann R, Pilarsky C, Izeradjene K, Hingorani SR, Huang P, Davies SE, Plunkett W, Egorin M, Hruban RH, Whitebread N, McGovern K, Adams J, Iacobuzio-Donahue C, Griffiths J, Tuveson DA (2009) Inhibition of Hedgehog signaling enhances delivery of chemotherapy in a mouse model of pancreatic cancer. Science 324(5933): 1457-1461.

Plunkett W, Huang P, Xu YZ, Heinemann V, Grunewald R, Gandhi V (1995) Gemcitabine: metabolism, mechanisms of action, and self-potentiation. Semin Oncol 22(4 Suppl 11): 3-10.

Rossi L, Serafini S, Schiavano GF, Casabianca A, Vallanti G, Chiarantini L, Magnani M (1999) Metabolism, mitochondrial uptake and toxicity of 2', 3'-dideoxycytidine. Biochem J 344(Pt 3): 915-920.

Tibaldi C, Giovannetti E, Tiseo M, Leon LG, D'Incecco A, Loosekoot N, Bartolotti M, Honeywell R, Cappuzzo F, Ardizzoni A, Peters GJ (2011) Correlation of cytidine deaminase polymorphisms and activity with clinical outcome in gemcitabine-/platinum-treated advanced non-smallcell lung cancer patients. Ann Oncol 23(3): 670-677.

Vaezian B, Anderton CR, Kraft ML (2010) Discriminating and imaging different phosphatidylcholine species within phase-separated model membranes by principal component analysis of TOF-secondary ion mass spectrometry images. Anal Chem 82(24): 10006-10014.

Veltkamp SA, Pluim D, van Eijndhoven MA, Bolijn MJ, Ong FH, Govindarajan R, Unadkat JD, Beijnen JH, Schellens JH (2008) New insights into the pharmacology and cytotoxicity of gemcitabine and 2',2'-difluorodeoxyuridine. Mol Cancer Ther 7(8): 2415-2425.

Von Hoff DD, Ervin T, Arena FP, Chiorean EG, Infante J, Moore M, Seay T, Tjulandin SA, Ma WW, Saleh MN, Harris M, Reni M, Dowden S, Laheru D, Bahary N, Ramanathan RK, Tabernero J, Hidalgo M, Goldstein D, Van Cutsem E, Wei X, Iglesias J, Renschler MF (2013) Increased survival in pancreatic cancer with nab-Paclitaxel plus gemcitabine. N Engl J Med 369(18): 1691-1703.

Workman P, Aboagye EO, Balkwill F, Balmain A, Bruder G, Chaplin DJ, Double JA, Everitt J, Farningham DA, Glennie MJ, Kelland LR, Robinson V, Stratford IJ, Tozer GM, Watson S, Wedge SR, Eccles SA. Committee of the National Cancer Research I (2010) Guidelines for the welfare and use of animals in cancer research. Br J Cancer 102(11): 1555-1577.

This work is licensed under the Creative Commons License. To view a copy of this license, visit http://creativecommons.
Lis org/licenses/by-nc-sa/3.0/

Supplementary Information accompanies this paper on British Journal of Cancer website (http://www.nature.com/bjc) 